\title{
Derivation of Arc Length of Helical Cable Element at Cable Bending, with Emphasize on Taylor Series Expansion of the Non-Integrable Infinitesimal Arc Length
}

\author{
Magnus Komperød ${ }^{1}$ \\ ${ }^{1}$ Technological Analyses Centre, Nexans Norway AS, Norway, magnus .komperod@nexans .com
}

\begin{abstract}
Elements of subsea cables and umbilicals can be classified as helical and non-helical. These two element types behave very differently at cable bending. This paper focuses on helical cable elements during cable bending. The arc length of helical elements at cable bending is derived, which leads to an integral that can not be solved analytically. When establishing strains and stresses of helical elements, it is essential that this integral is calculated with very high accuracy. An integration error of $0.01 \%$ is unacceptable in many applications. Maclaurin series expansion is used to convert this integral into an integral that can be solved analytically. It is proved that arbitrary integration accuracy can be achieved by increasing the order of the Maclaurin polynomial. Expressions for upper and lower boundaries of the integration error are also provided. The main advantage of the integration approach derived in this paper is that it provides an analytical expression for the integral, which can be used in mechanical analyses of cables and umbilicals.
\end{abstract}

Keywords: Analytical Integration; Cross Section Analysis; Helical Cable Element; Maclaurin Series Expansion; Offshore Technology; Subsea Cable; Taylor's Theorem; Umbilical.

\section{Introduction}

Subsea power cables are essential for overseas power transmission, while umbilicals are necessary for offshore oil and gas production. Umbilicals are cable-like structures that consist of different types of elements, such as tubes for hydraulic equipment and transportation of fluids, electric and fiber optic signal cables, and electric power phases for energizing subsea units.

The elements of subsea cables and umbilicals can be categorized as helical elements and non-helical elements. Non-helical elements are parallel to the cable's length direction. Common non-helical elements are the cable's outer sheath and possible center tube or center profile.

Helical elements form helices which helix centers are equivalent to the cable's cross section center. Helical elements are typically power phases, tubes, electric and fiber optic signal cables, as well as armor wires. Figure 1 shows an umbilical with a non-helical center tube, two layers of helical tubes, and one layer consisting of helical tubes, helical electric signal cables, and a helical fiber optic signal cable. The umbilical also has four layers of helical steel armor wires. The yellow outer sheath is non-helical.

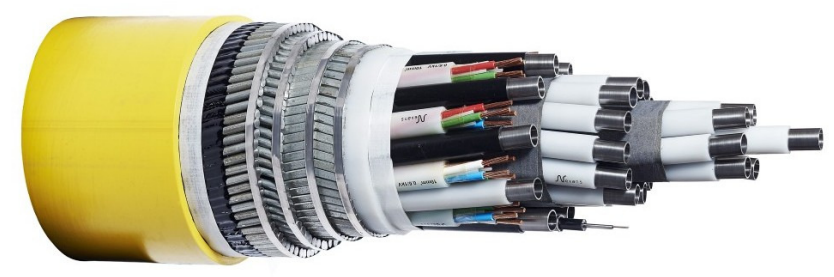

Figure 1. Umbilical with helical tubes, helical electric and fiber optic signal cables, and helical armor wires.

Many cable elements, in particular metallic elements, have mechanical capacity criteria that are not to be exceeded during cable handling, installation, and operation. For metallic elements, these criteria are typically a certain percentage of the elements' respective yield limits. In terms of not exceeding the capacity criteria, helical elements behave more favorably during cable bending than non-helical elements. During cable bending, non-helical cable elements are inherently tensioned at the outer arc and compressed at the inner arc. Helical cable elements can slide inside the cable in such a way that the element's tension at the cable's outer arc are canceled by the element's compression at the cable's inner arc, only limited by possible shear forces, such as friction.

Modeling of helical elements during bending of cables and similar structures are covered in the literature. For example Féret and Bournazel (1987), Kebadze (2000), Sødahl et al. (2010), and Skeie et al. (2012) discuss bending 
of cables and similar structures not constrained by end effects. Sævik (1992) and Komperød et al. (2015) discuss bending of cable segments that are confined by end terminations, while Lutchansky (1969) considers stresses in helical elements close to cable clamps.

Strains and stresses of helical elements are essential for modeling the mechanical properties of cables, such as axial stiffness, bending stiffness, torsion stiffness, and capacity (allowed combinations of axial cable tension and cable bending curvature). To obtain correct values for strains and stresses, it is essential to calculate the arc length of the helical elements with very high accuracy. A length error of $0.01 \%$ is unacceptable in many cases.

How to calculate the arc length of helical elements in bent cables is derived in the literature, see for example Lutchansky (1969) and Komperød et al. (2015). These derivations lead to an integral that can not be solved analytically. The contribution of the present paper is to use Maclaurin series expansion to rewrite this integral into integrals that can be solved analytically. Sævik (1992) points out that using too few terms of the Maclaurin series may lead to inaccurate results.

The present paper proves mathematically that arbitrary accuracy of the integral can be achieved by increasing the order of the Maclaurin polynomial. It is also derived expressions for upper and lower boundaries that the true element length is ensured to be within. These boundaries narrow as the polynomial order increases. Finally, the proposed method is demonstrated through a numerical example. In the example, the Maclaurin approach is compared to numeric integration using Simpson's method. The latter method also serves the purpose of verifying the former method. The main advantage of the Maclaurin approach, compared to Simpson's method, is that the Maclaurin approach provides an analytical expression for the integral, which can be used in mechanical analyses of cables and umbilicals.

\section{Nomenclature}

Table 1 presents the nomenclature used in this paper.

\section{Assumptions and Simplifications}

The mathematical derivation of this paper is subject to the follow assumptions and simplifications:

1. The helical cable elements are assumed to follow loxodromic curves at cable bending. This means that if a helical element slips the beneath surface, it slips parallel to its tangent vector.

The loxodromic approach is one of three common approaches in the literature. Another approach is to assume the helical elements to follow a geodesic
Table 1. Nomenclature.

\begin{tabular}{|c|c|}
\hline$c$ & Variable defined in Eq. 13 [-]. \\
\hline$k$ & Exponent [-]. \\
\hline$L$ & Pitch length [m]. \\
\hline$l$ & Cable length [m]. \\
\hline$N$ & Order of Maclaurin polynomial [-]. \\
\hline$P_{N}(x)$ & Maclaurin polynomial of order $N$ [-]. \\
\hline$Q_{N}(x)$ & $\begin{array}{l}\text { Reminder of Maclaurin polynomial of } \\
\operatorname{order} N[-] \text {. }\end{array}$ \\
\hline$Q_{N}^{\text {lower }}(x)$ & Lower boundary of $Q_{N}(x)[-]$ \\
\hline$Q_{N}^{\text {upper }}(x)$ & Upper boundary of $Q_{N}(x)[-]$. \\
\hline $\bar{Q}_{N}(x)$ & Variable defined in Eq. 23 [-]. \\
\hline $\bar{Q}_{N}^{\max }(x)$ & Maximum value of $\bar{Q}_{N}(x)$ [-]. \\
\hline$R$ & Pitch radius [m]. \\
\hline$s$ & Length of helical cable element [m]. \\
\hline$s\left(\theta_{1}, \theta_{2}\right)$ & $\begin{array}{l}\text { Length of helical cable element from } \theta_{1} \\
\text { to } \theta_{2}[\mathrm{~m}] \text {. }\end{array}$ \\
\hline$s_{\mathrm{a}}$ & Axial component of $s[\mathrm{~m}]$ \\
\hline$s_{\mathrm{h}}$ & Circumference component of $s[\mathrm{~m}]$. \\
\hline$x$ & Variable defined in Eq. $8[-]$. \\
\hline$x^{\min }$ & $\begin{array}{l}\text { Minimum value of } x \text { on interval }\left[\theta_{1}, \theta_{2}\right] \\
{[-] \text {. }}\end{array}$ \\
\hline$x^{\max }$ & $\begin{array}{l}\text { Maximum value of } x \text { on interval }\left[\theta_{1}, \theta_{2}\right] \\
{[-] .}\end{array}$ \\
\hline $\bar{x}$ & Variable defined in Eq. 20 [-]. \\
\hline$\alpha$ & Pitch angle [rad]. \\
\hline$\theta$ & Angle in cable's cross section [rad]. \\
\hline$\theta_{1}$ & Lower integration limit [rad]. \\
\hline$\theta_{2}$ & Upper integration limit [rad]. \\
\hline$\kappa$ & Cable bending curvature $\left[\mathrm{m}^{-1}\right]$. \\
\hline
\end{tabular}

curve during cable bending. In the geodesic approach, the helical element slips both parallel and perpendicular to its tangent vector. The third approach is to assume the helical elements to follow a curve that is between the loxodromic curve and the geodesic curve. Please refer to Sævik (1992) for an explanation of loxodromic curves and geodesic curves at cable bending.

2. Displacements of helical cable elements in the cable's radial direction are neglected.

3. The cable is assumed not to twist around its length axis.

4. The cable's path is assumed to have zero torsion, i.e. the cable is assumed to be bent in a two-dimensional plane, not a three-dimensional room. 
5. The curvature, $\kappa$, may be a constant, or it may be a function of $\theta$, i.e. $\kappa=\kappa(\theta)$. In the latter case, the function $\kappa(\theta)$ must be on a form such that $x^{n}$ is analytically integrable, where $x$ is defined in Eq. 8 and $n$ is a nonnegative integer.

\section{Derivation of the Infinitesimal Arc Length}

This section derives the infinitesimal arc length of a helical element in a bent cable. The derivation is strongly inspired by Komperød et al. (2015). Lutchansky (1969) presents a somewhat different derivation, which yields identical result. The derivation of Lutchansky (1969) is longer and somewhat more complex, but it has the advantage of also deriving a parameterization of the helical element, which is not the case for Komperød et al. (2015).

Let $l$ be the length of the cable, and let $s$ be the length of a helical cable element. Over an infinitesimal cable length, $\mathrm{d} l$, the cable and its elements follow their respective tangent vectors. Hence, $\mathrm{d} l$ and $\mathrm{d} s$ are straight lines. $\mathrm{d} s$ can be decomposed into two components; $\mathrm{d} s_{\mathrm{a}}$ that is parallel to $\mathrm{d} l$, and $\mathrm{d} s_{\mathrm{h}}$ that is perpendicular to $\mathrm{d} l$. Figure 2 illustrates the relationship between the lengths $l, s, s_{\mathrm{a}}$, and $s_{\mathrm{h}}$. The lengths $\mathrm{d} s, \mathrm{~d} s_{\mathrm{a}}$, and $\mathrm{d} s_{\mathrm{h}}$ are related through Pythagoras' theorem

$$
\mathrm{d} s=\sqrt{\mathrm{d} s_{\mathrm{a}}^{2}+\mathrm{d} s_{\mathrm{h}}^{2}}
$$

By definition, the center of a helical element is a radial distance $R$ from the cable's cross section center, where $R$ is the element's pitch radius. The angle $\theta$ defines the helical element's position in the cable cross section: For positive bending curvature, i.e. $\kappa>0$, the helical element is at the cable's outer arc for $\theta=\frac{\pi}{2}$, and at the inner arc for $\theta=$ $-\frac{\pi}{2}$. For negative bending curvature, i.e. $\kappa<0$, the helical

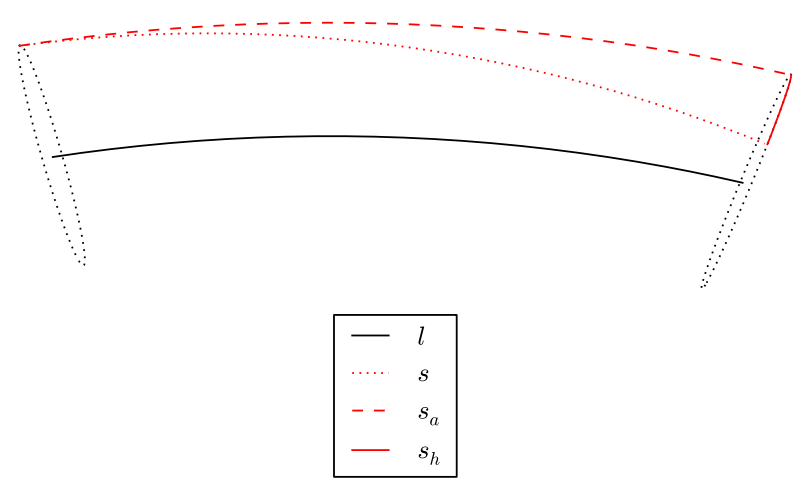

Figure 2. Relationship between $l, s, s_{\mathrm{a}}$, and $s_{\mathrm{h}}$. element is at the cable's outer arc for $\theta=-\frac{\pi}{2}$, and at the inner arc for $\theta=\frac{\pi}{2}$. The helical element is at the cable's neutral axis for $\theta=0$ and for $\theta=\pi$, regardless of the sign of $\kappa$. By definition, $\theta$ increases $2 \pi$ for each pitch length, $L$, along the cable's length, $l$. That is, $\theta$ and $l$ are related through

$$
\frac{\theta}{l}=\frac{2 \pi}{L}
$$

Over an infinitesimal cable length, $\mathrm{d} l$, the cable's cross section center follows a circle arc with radius $\frac{1}{\kappa}$. Because the cable's cross section center is a radial distance $\frac{1}{\kappa}$ from the center of the circle arc, the helical element's radial distance is $\frac{1}{\kappa}+R \sin (\theta)$. Hence, $s_{\mathrm{a}}$ is related to $l$ through the relation

$$
\begin{aligned}
\mathrm{d} s_{\mathrm{a}} & =\frac{\frac{1}{\kappa}+R \sin (\theta)}{\frac{1}{\kappa}} \mathrm{d} l \\
& =(1+R \kappa \sin (\theta)) \mathrm{d} l .
\end{aligned}
$$

From Eq. 2 it follows that over a cable length $\mathrm{d} l$, the helical cable element completes an angle $\mathrm{d} \theta=\frac{2 \pi}{L} \mathrm{~d} l$. Because the element is located a distance $R$ from the cable's cross section center, the angle $\mathrm{d} \theta$ corresponds to an arc length in circumference direction of $\mathrm{d} s_{\mathrm{h}}=R \mathrm{~d} \theta$. Hence,

$$
\mathrm{d} s_{\mathrm{h}}=\frac{2 \pi R}{L} \mathrm{~d} l .
$$

Inserting Eq. 3 and Eq. 4 into Eq. 1 gives the relationship between the helical cable element's length, $s$, and the cable's length, $l$, as

$$
\mathrm{d} s=\sqrt{(1+R \kappa \sin (\theta))^{2}+\left(\frac{2 \pi R}{L}\right)^{2}} \mathrm{~d} l
$$

The desired result is the length of the helical element, $s$, from $\theta_{1}$ to $\theta_{2}$, i.e. the integral of Eq. 5, which is

$$
s\left(\theta_{1}, \theta_{2}\right)=\int_{\frac{L}{2 \pi} \theta_{1}}^{\frac{L}{2 \pi} \theta_{2}} \sqrt{(1+R \kappa \sin (\theta))^{2}+\left(\frac{2 \pi R}{L}\right)^{2}} \mathrm{~d} l,
$$

where the integration limits follow from Eq. 2. Please note that the square root of Eq. 6 can not be considered a constant during integration, because $\theta$ depends on $l$. The exceptions are the two special cases of $R=0$ and $\kappa=0$, where the square root expression is independent of $l$ and can hence be considered a constant during integration. The physical interpretation of $R=0$ is that the element is not a helical element, but a non-helical element 
located in the cable's cross section center. The case $\kappa=0$ means that the cable is straight (not bent).

To the author's knowledge, it is not possible to integrate Eq. 6 analytically. The main contribution of the present paper is to approximate the integrand using Maclaurin series expansion, and then to estimate the integration error of this approach.

\section{Maclaurin Series Expansion}

Using Eq. 2 and the relationship $\tan (\alpha)=\frac{2 \pi R}{L}$, where $\alpha$ is the element's pitch angle, Eq. 5 can be rewritten to

$$
\mathrm{d} s=\frac{R}{\sin (\alpha)}(1+x)^{\frac{1}{2}} \mathrm{~d} \theta
$$

where

$$
x \stackrel{\text { def }}{=} \cos ^{2}(\alpha)\left[2 R \kappa \sin (\theta)+R^{2} \kappa^{2} \sin ^{2}(\theta)\right]
$$

The term $(1+x)^{\frac{1}{2}}$ of Eq. 7 is recognized from the theory of binomial series. That is

$$
(1+x)^{k}=1+\sum_{n=1}^{\infty}\left(\begin{array}{l}
k \\
n
\end{array}\right) x^{n}
$$

where the right hand side is the Maclaurin series of the left hand side, and where

$$
\left(\begin{array}{l}
k \\
n
\end{array}\right) \stackrel{\text { def }}{=} \frac{k(k-1)(k-2) \ldots(k-n+1)}{n !} .
$$

\subsection{Proof of Convergence and Estimation of Reminder}

When inserting Eq. 8 into Eq. 9, and then inserting Eq. 9 into Eq. 7, the right hand side of Eq. 7 becomes an infinite series of which each term can be integrated analytically w.r.t. $\theta$. However, it is not feasible to sum infinite many terms. Hence, the series of Eq. 9 must be truncated before it is inserted into Eq. 7.

This section uses Taylor's theorem to prove that the right hand side of Eq. 9 converges to the left hand side, and to estimate the reminder (approximation error). In order to use Taylor's theorem, the constraint $-\frac{1}{2}<x<1$ must be imposed. For subsea cables and umbilicals, $R$ is rarely more than $0.2 \mathrm{~m}$, and $\kappa$ is rarely more than $0.5 \mathrm{~m}^{-1}$. The absolute value of $x$ is not likely to exceed 0.2 , or at least not 0.3 . Therefore, the imposed constraint is unlikely to be an issue for real-life cables.

Please note that according to the binomial theorem, the right hand side of Eq. 9 converges to the left hand side for $|x|<1$ (ProofWiki, 2015). Hence, the convergence proof of this section is actually superfluous. However, proving convergence using Taylor's theorem also provides a reminder estimate which is essential for Section 6.

For two variables $a$ and $x$ in an open interval, in which $f(x)$ and its first $N+1$ derivatives are continuous, Taylor's theorem states that

$$
f(x)=P_{N}(x)+Q_{N}(x),
$$

where

$$
\begin{aligned}
& P_{N}(x)=f(a)+\sum_{n=1}^{N} \frac{\left.\frac{\mathrm{d}^{n} f(x)}{\mathrm{d} x^{n}}\right|_{x=a}}{n !}(x-a)^{n}, \\
& Q_{N}(x)=\frac{\left.\frac{\mathrm{d}^{N+1} f(x)}{\mathrm{d} x^{N+1}}\right|_{x=c}}{(N+1) !}(x-a)^{N+1},
\end{aligned}
$$

for some $c$, where $x<c<a$ if $x<a$, and $a<c<x$ if $x>a$ (Thomas et al., 2010). In Eq. 11, $P_{N}$ is the Taylor polynomial of order $N$, and $Q_{N}$ is the reminder of this polynomial.

Inserting $f(x)=(1+x)^{k}$ and $a=0$ into Eqs. 11-13, gives

$$
(1+x)^{k}=P_{N}(x)+Q_{N}(x),
$$

where

$$
\begin{aligned}
& P_{N}(x)=1+\sum_{n=1}^{N}\left(\begin{array}{l}
k \\
n
\end{array}\right) x^{n}, \\
& Q_{N}(x)=\left(\begin{array}{c}
k \\
N+1
\end{array}\right)(1+c)^{k-N-1} x^{N+1},
\end{aligned}
$$

$x<c<0$ if $x<0$, and $0<c<x$ if $x>0$. Proving that $P_{N}$ converges to $(1+x)^{k}$, i.e.

$$
\lim _{N \rightarrow \infty} P_{N}(x)=(1+x)^{k},
$$

is done by proving that $Q_{N}$ goes to zero, i.e.

$$
\lim _{N \rightarrow \infty} Q_{N}(x)=0 .
$$

Eq. 18 will now be proved. Eq. 16 can be rewritten to

$$
Q_{N}(x)=\left(\begin{array}{c}
k \\
N+1
\end{array}\right) x(1+c)^{k-1} \bar{x}^{N},
$$




$$
\bar{x} \stackrel{\text { def }}{=} \frac{x}{1+c} .
$$

By the constraints imposed on $x$ and $c$

$$
\frac{-\frac{1}{2}}{1+\left(-\frac{1}{2}\right)}<\bar{x}<\frac{1}{1+0}
$$

which gives

$$
|\bar{x}|<1
$$

For $k-1>0$ the maximum value of $(1+c)^{k-1}$ in Eq. 19 is less than $2^{k-1}$. For $k-1<0$ the maximum value of $(1+c)^{k-1}$ is less than $\left(1+\left(-\frac{1}{2}\right)\right)^{k-1}=2^{|k-1|}$. Hence, regardless of $k$, the maximum value of $(1+c)^{k-1}$ is less than $2^{|k-1|}$.

The sequence $\bar{Q}_{N}(x)$ is defined as

$$
\bar{Q}_{N}(x) \stackrel{\text { def }}{=}\left|\left(\begin{array}{c}
k \\
N+1
\end{array}\right) 2^{|k-1|} \bar{x}^{N}\right| .
$$

Then

$$
-\bar{Q}_{N}(x) \leq Q_{N}(x) \leq \bar{Q}_{N}(x)
$$

The limit of $\bar{Q}_{N}(x)$ can be established through the relation

$$
\begin{aligned}
\lim _{N \rightarrow \infty} \frac{\bar{Q}_{N+1}(x)}{\bar{Q}_{N}(x)} & =\lim _{N \rightarrow \infty} \frac{\left.\mid \begin{array}{c}
k \\
N+2
\end{array}\right) 2^{|k-1|} \bar{x}^{N+1} \mid}{\left.\mid \begin{array}{c}
k \\
N+1
\end{array}\right) 2^{|k-1|} \bar{x}^{N} \mid} \\
& =\lim _{N \rightarrow \infty}\left|\frac{k-N-1}{N+2} \bar{x}\right| \\
& =|\bar{x}| .
\end{aligned}
$$

The result of Eq. 25 states that as $N \rightarrow \infty, \bar{Q}_{N}(x)$ will decrease by a factor $|\bar{x}|$ for each increment of $N$. Hence,

$$
\lim _{N \rightarrow \infty}-\bar{Q}_{N}(x)=\lim _{N \rightarrow \infty} \bar{Q}_{N}(x)=0
$$

Using the sandwich theorem for sequences (Thomas et al., 2010, Theorem 2 p. 536), Eqs. 24 and 26 prove Eq. 18. Taking the limit $N \rightarrow \infty$ of Eq. 14 and inserting Eq. 18 proves Eq. 17. This means that as the number of terms in the Maclaurin polynomial, $P_{N}(x)$, increases, the polynomial approaches $(1+x)^{k}$ and the reminder, $Q_{N}(x)$, approaches zero. For a finite number of terms, the polynomial and the reminder are given by Eqs. 15 and 16, respectively.
Inserting Eqs. 14-16 into Eq. 7 and setting $k=\frac{1}{2}$ gives the infinitesimal length of the helical element, $\mathrm{d} s$

$$
\begin{aligned}
\mathrm{d} s= & \frac{R}{\sin (\alpha)}\left[1+\sum_{n=1}^{N}\left(\begin{array}{c}
\frac{1}{2} \\
n
\end{array}\right) x^{n}\right] \mathrm{d} \theta \\
& +\frac{R}{\sin (\alpha)}\left(\begin{array}{c}
\frac{1}{2} \\
N+1
\end{array}\right)(1+c)^{-N-\frac{1}{2}} x^{N+1} \mathrm{~d} \theta
\end{aligned}
$$

where $x$ is given by Eq. 8. The upper right hand side of Eq. 27 is the Maclaurin polynomial, while the lower right hand side is the reminder of this polynomial.

\subsection{Numerical Example of the Maclaurin Polymonial's Accuracy}

Figure 3 shows the true value for $\frac{R}{\sin (\theta)}(1+x)^{\frac{1}{2}}$ of Eq. 7 plotted against the corresponding Maclaurin polynomials of orders 1 through 4 for the numerical values stated in Table 2. To the resolution of the figure, only the first order Maclaurin polynomial can be distinguished from the true value. Figure 4 shows the same graphs zoomed in at the maxima. This figure visualizes well the decrease rate of the approximation error as the polynomial order increases. The forth order polynomial approximates the true value so well that they can not be distinguished even in the zoomed figure.

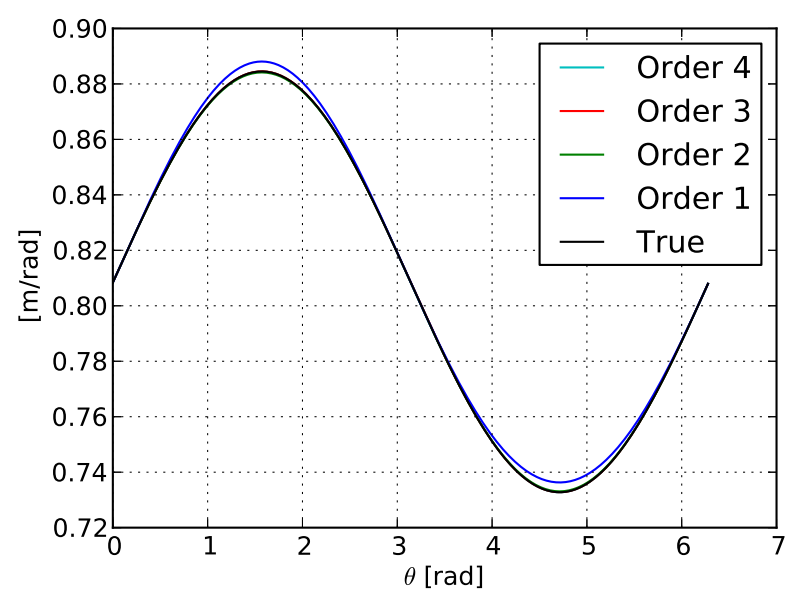

Figure 3. Comparing $\frac{R}{\sin (\theta)}(1+x)^{\frac{1}{2}}$ with Maclaurin polynomials of orders 1 through 4 .

Table 2. Values used in numerical examples.

\begin{tabular}{lrl}
\hline Variable & Value & Unit \\
\hline$\alpha$ & 0.25 & $\mathrm{rad}$ \\
$R$ & 0.20 & $\mathrm{~m}$ \\
$\kappa$ & 0.50 & $\mathrm{~m}^{-1}$ \\
\hline
\end{tabular}




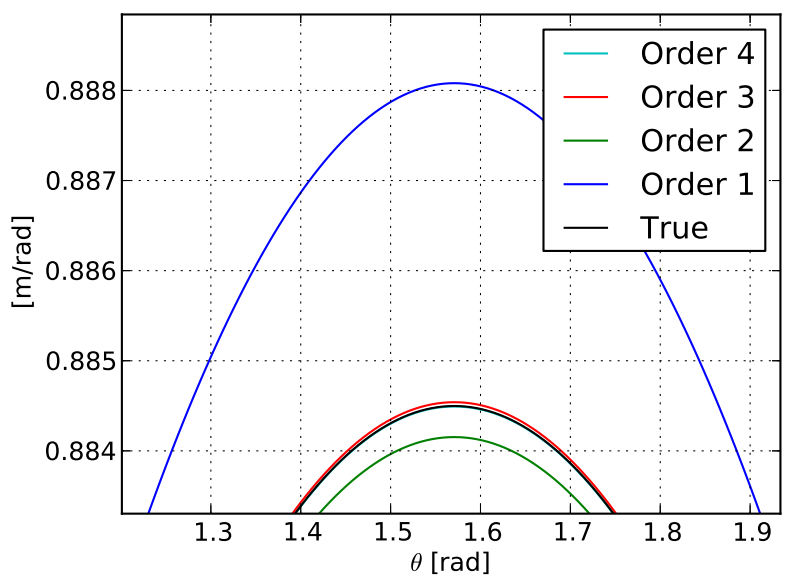

Figure 4. Comparing $\frac{R}{\sin (\theta)}(1+x)^{\frac{1}{2}}$ with Maclaurin polynomials of orders 1 through 4 , zoomed in close to the maxima.

\section{Integration of the Maclaurin Poly- nomial}

This section considers analytical integration of the Maclaurin polynomial. This integral is an approximation of Eq. 6, which can not be integrated analytically.

\subsection{Proof of Convergence after Integration}

In this section it is proved that arbitrary accuracy of the helical cable element's length, $s\left(\theta_{1}, \theta_{2}\right)$, can be achieved by increasing the order of the Maclaurin polynomial, $P_{N}(x)$.

Inserting Eq. 14 into Eq. 7 and integrating w.r.t. $\theta$ from $\theta_{1}$ to $\theta_{2}$, where $\theta_{2}>\theta_{1}$, gives

$$
\begin{aligned}
s\left(\theta_{1}, \theta_{2}\right)= & \int_{\theta_{1}}^{\theta_{2}} \frac{R}{\sin (\alpha)} P_{N}(x) \mathrm{d} \theta \\
& +\int_{\theta_{1}}^{\theta_{2}} \frac{R}{\sin (\alpha)} Q_{N}(x) \mathrm{d} \theta .
\end{aligned}
$$

It will now be proved that the upper right term converges to the length of the helical cable element, $s\left(\theta_{1}, \theta_{2}\right)$, by proving that the lower right term converges to zero. $\bar{Q}_{N}^{\max }$ is defined as

$$
\bar{Q}_{N}^{\max } \stackrel{\text { def }}{=} \underset{\theta}{\arg \max } \bar{Q}_{N}
$$

Eq. 24 can then be extended to

$$
-\bar{Q}_{N}^{\max } \leq-\bar{Q}_{N}(x) \leq Q_{N}(x) \leq \bar{Q}_{N}(x) \leq \bar{Q}_{N}^{\max }
$$

Hence,

$$
-\bar{Q}_{N}^{\max } \leq Q_{N}(x) \leq \bar{Q}_{N}^{\max }
$$

Multiplying Eq. 31 with the non-negative factor $\frac{R}{\sin (\alpha)}$ gives

$$
\begin{aligned}
-\frac{R}{\sin (\alpha)} \bar{Q}_{N}^{\max }(x) & \leq \frac{R}{\sin (\alpha)} Q_{N}(x) \\
& \leq \frac{R}{\sin (\alpha)} \bar{Q}_{N}^{\max }(x)
\end{aligned}
$$

As this inequality holds for any $\theta$, the inequality will also hold after taking the Riemann sum w.r.t. $\theta$ of each expression. As integration can be considered taking the norm limit of the Riemann sum, the inequality will also hold after integration from $\theta_{1}$ to $\theta_{2}$, where $\theta_{2}>\theta_{1}$, i.e.

$$
\begin{aligned}
-\int_{\theta_{1}}^{\theta_{2}} \frac{R}{\sin (\alpha)} \bar{Q}_{N}^{\max }(x) \mathrm{d} \theta & \leq \int_{\theta_{1}}^{\theta_{2}} \frac{R}{\sin (\alpha)} Q_{N}(x) \mathrm{d} \theta \\
& \leq \int_{\theta_{1}}^{\theta_{2}} \frac{R}{\sin (\alpha)} \bar{Q}_{N}^{\max }(x) \mathrm{d} \theta
\end{aligned}
$$

Because $\bar{Q}_{N}^{\max }$ by definition is the largest value of $\bar{Q}_{N}$ for any $\theta, \bar{Q}_{N}^{\max }$ is not a function of $\theta$. Hence, Eq. 33 can be rewritten

$$
\begin{aligned}
& -\frac{R}{\sin (\alpha)} \bar{Q}_{N}^{\max }(x)\left(\theta_{2}-\theta_{1}\right) \\
& \leq \int_{\theta_{1}}^{\theta_{2}} \frac{R}{\sin (\alpha)} Q_{N}(x) \mathrm{d} \theta \\
& \leq \frac{R}{\sin (\alpha)} \bar{Q}_{N}^{\max }(x)\left(\theta_{2}-\theta_{1}\right) .
\end{aligned}
$$

Eq. 26 holds for any $\theta$. Hence,

$$
\lim _{N \rightarrow \infty}-\bar{Q}_{N}^{\max }(x)=\lim _{N \rightarrow \infty} \bar{Q}_{N}^{\max }(x)=0 .
$$

Taking the limit of Eq. 34 and inserting Eq. 35 then gives

$$
0 \leq \lim _{N \rightarrow \infty} \int_{\theta_{1}}^{\theta_{2}} \frac{R}{\sin (\alpha)} Q_{N}(x) \mathrm{d} \theta \leq 0
$$

Then by the sandwich theorem for sequences it is proved that

$$
\lim _{N \rightarrow \infty} \int_{\theta_{1}}^{\theta_{2}} \frac{R}{\sin (\alpha)} Q_{N}(x) \mathrm{d} \theta=0
$$


Taking the limit $N \rightarrow \infty$ of Eq. 28 and inserting Eq. 37 proves that

$$
s\left(\theta_{1}, \theta_{2}\right)=\lim _{N \rightarrow \infty} \int_{\theta_{1}}^{\theta_{2}} \frac{R}{\sin (\alpha)} P_{N}(x) \mathrm{d} \theta .
$$

The practical interpretation of Eq. 38 is that arbitrary accuracy of the helical cable element's length, $s\left(\theta_{1}, \theta_{2}\right)$, can be achieved by increasing the order of the Maclaurin polymomial, $P_{N}(x)$.

\subsection{Advantages of the Maclaurin Polynomial Approach}

The main motivation for the work presented in this paper is to obtain an analytical expression for the length of helical cable elements at cable bending, i.e. an analytical solution to the integral of Eq. 6. This analytical solution can be used in mechanical analyses of cables and umbilicals. The Maclaurin approach proposed in this paper also has some interesting properties w.r.t. integration accuracy which are discussed in this section.

Inserting Eq. 15 into Eq. 38 and solving the integral of the constant term of $P_{N}(x)$ gives

$$
\begin{aligned}
s\left(\theta_{1}, \theta_{2}\right)= & \frac{R}{\sin (\alpha)}\left(\theta_{2}-\theta_{1}\right) \\
& +\lim _{N \rightarrow \infty} \frac{R}{\sin (\alpha)}\left[\sum_{n=1}^{N}\left(\begin{array}{l}
k \\
n
\end{array}\right) \int_{\theta_{1}}^{\theta_{2}} x^{n} \mathrm{~d} \theta\right] .
\end{aligned}
$$

When the cable is straight (not bent), i.e. when $\kappa=0, x$ vanishes. Then the lower right term of Eq. 39 vanishes. Hence, the upper right term is the helical cable element's length when the cable is straight, and the lower right term is the element's elongation as the cable is bent. Negative elongation means compression.

For realistic values of $R$ and $\kappa$, the helical cable element's elongation or compression due to cable bending is very small compared to the element's initial length. From Eq. 39 it then follows

$$
\begin{gathered}
\left|\lim _{N \rightarrow \infty} \frac{R}{\sin (\alpha)}\left[\sum_{n=1}^{N}\left(\begin{array}{l}
k \\
n
\end{array}\right) \int_{\theta_{1}}^{\theta_{2}} x^{n} \mathrm{~d} \theta\right]\right| \\
\ll \frac{R}{\sin (\alpha)}\left(\theta_{2}-\theta_{1}\right) \approx s\left(\theta_{1}, \theta_{2}\right) .
\end{gathered}
$$

It may be tempting to neglect the upper term of Eq. 40 for the purpose of simplifying the equation. However, that would be a very serious mistake, because it is this elongation, although very small, that causes the strains and stresses on which the mechanical analyses are based.
An intuitive way to establish the helical cable element's elongation due to cable bending is to integrate Eq. $6 \mathrm{nu}-$ merically for bent cable and then subtract the element's length at straight cable. As the element's length changes only slightly during cable bending, the numerical integration must be very accurate, otherwise erroneous results are reached. In many cases an integration error of $0.01 \%$ will be unacceptable.

The Maclaurin approached presented in this paper has the advantage of analytically separating the helical cable element's length before cable bending (the upper right term of Eq. 39) and the element's elongation or compression due to cable bending (the lower right term of Eq. 39). Further, the Maclaurin approach has the benefit that each term can be integrated analytically, which nearly eliminates numerical errors. However, some error due to truncation of the Maclaurin series is inherent in the proposed method. An approach to calculate this error is presented in Section 6.3.

As will be shown in Section 6.4, numeric integration using Simpson's method also gives very good results.

\subsection{Estimation of Error}

Section 6.1 proved Eq. 38, with the practical interpretation that arbitrary accuracy of the helical cable element's length, $s\left(\theta_{1}, \theta_{2}\right)$, can be achieved by increasing the order of the Maclaurin polynomial, $P_{N}(x)$. The present section considers how to estimate the approximation error of $s\left(\theta_{1}, \theta_{2}\right)$ due to truncation of $P_{N}(x)$ at a finite polynomial order, $N$. The error is given from Eq. 28 as

$$
\begin{aligned}
\int_{\theta_{1}}^{\theta_{2}} \frac{R}{\sin (\alpha)} Q_{N}(x) \mathrm{d} \theta= & s\left(\theta_{1}, \theta_{2}\right) \\
& -\int_{\theta_{1}}^{\theta_{2}} \frac{R}{\sin (\alpha)} P_{N}(x) \mathrm{d} \theta .
\end{aligned}
$$

In other words, the purpose of this section is how to estimate the left hand side of Eq. 41, where $Q_{N}(x)$ is given by Eq. 16. Please note that it is not straight forward to integrate the left hand side of Eq. 41 , because $Q_{N}(x)$ contains the variable $c$, which is unknown and varies with $x$. There are several ways to estimate this integral, with different degrees of accuracy, and different degrees of complexity and difficulty. The method proposed below is believed to be a feasible compromise between accuracy and complexity.

The basic principle of the proposed method is to provide upper and lower limits of $Q_{N}(x)$, which are sufficiently close to the true $Q_{N}(x)$ to be reasonable accurate, while being simple enough to be integrated analytically.

Define $x^{\min }$ and $x^{\max }$ as the lowest and highest values, respectively, of $x$ within (and including) the integration limits $\theta_{1}$ and $\theta_{2}$. That is 


$$
\begin{gathered}
x^{\min } \stackrel{\text { def }}{=} \underset{\theta \in\left[\theta_{1}, \theta_{2}\right]}{\arg } \min x, \\
x^{\max } \stackrel{\text { def }}{=} \underset{\theta \in\left[\theta_{1}, \theta_{2}\right]}{\arg \max x .}
\end{gathered}
$$

For $k=\frac{1}{2}$, Eq. 16 can be written as

$$
Q_{N}(x)=\left(\begin{array}{c}
\frac{1}{2} \\
N+1
\end{array}\right) x^{N+1} \frac{1}{(1+c)^{N+\frac{1}{2}}}
$$

The binomial factor, $\left(\begin{array}{c}\frac{1}{2} \\ N+1\end{array}\right)$, is positive for even $N$, and negative for odd $N$. As $c>-\frac{1}{2}$, the factor $\frac{1}{(1+c)^{N+\frac{1}{2}}}$ is always positive. The factor $x^{N+1}$ is negative when $N$ is even and $x<0$, otherwise positive.

All values on the right hand side of Eq. 44 are known, except for $c$. While $c$ is unknown, it is known to be within the intervals stated in Section 5.1. That is $x^{\min } \leq x<c<0$ if $x<0$, and $0<c<x \leq x^{\max }$ if $x>0$, where the inequalities have been extended by using Eqs. 42 and 43 .

Let $Q_{N}^{\text {lower }}(x)$ and $Q_{N}^{\text {upper }}(x)$ be lower and upper boundaries, respectively, that $Q_{N}(x)$ always will be within provided that the previously stated constraints are obeyed. In other words, the following inequality always holds:

$$
Q_{N}^{\text {lower }}(x) \leq Q_{N}(x) \leq Q_{N}^{\text {upper }}(x) .
$$

Subject to the constraints imposed on $c$, it can be shown that

$$
\frac{\partial}{\partial c} \frac{1}{(1+c)^{N+\frac{1}{2}}}<0 .
$$

This inequality, together with the constrains of $c$, will be used to derive expressions for $Q_{N}^{\text {lower }}(x)$ and $Q_{N}^{\text {upper }}(x)$. The derivation must be divided into four cases, depending on whether $N$ is odd or even, and on whether $x$ is positive or negative:

\section{$N$ is Even, $x$ is Positive}

If $N$ is even and $x>0$, then $\left(\begin{array}{c}\frac{1}{2} \\ N+1\end{array}\right) x^{N+1}>0$. From Eq. 46 it then follows that

$$
\frac{\partial}{\partial c}\left[\left(\begin{array}{c}
\frac{1}{2} \\
N+1
\end{array}\right) x^{N+1} \frac{1}{(1+c)^{N+\frac{1}{2}}}\right]<0 .
$$

As the derivative is negative, the lower limit, $Q_{N}^{\text {lower }}$, is found by inserting the highest value for $c$, and the upper limit, $Q_{N}^{\text {upper }}$, is found by inserting the lowest value of $c$. Because $x>0, c$ is constrained by $0<c<x^{\max }$. Hence,

$$
\begin{aligned}
& Q_{N}^{\text {lower }}(x)=\left(\begin{array}{c}
\frac{1}{2} \\
N+1
\end{array}\right) x^{N+1} \frac{1}{\left(1+x^{\max }\right)^{N+\frac{1}{2}}}, \\
& Q_{N}^{\text {upper }}(x)=\left(\begin{array}{c}
\frac{1}{2} \\
N+1
\end{array}\right) x^{N+1} .
\end{aligned}
$$

\section{$N$ is Even, $x$ is Negative}

If $N$ is even and $x<0$, then $\left(\begin{array}{c}\frac{1}{2} \\ N+1\end{array}\right) x^{N+1}<0$. That gives,

$$
\frac{\partial}{\partial c}\left[\left(\begin{array}{c}
\frac{1}{2} \\
N+1
\end{array}\right) x^{N+1} \frac{1}{(1+c)^{N+\frac{1}{2}}}\right]>0 .
$$

As the derivative is positive, the lower limit is found by inserting the lowest value for $c$, and vice versa. Because $x<0, c$ is constrained by $x^{\min }<c<0$. Hence,

$$
\begin{aligned}
& Q_{N}^{\text {lower }}(x)=\left(\begin{array}{c}
\frac{1}{2} \\
N+1
\end{array}\right) x^{N+1} \frac{1}{\left(1+x^{\text {min }}\right)^{N+\frac{1}{2}}}, \\
& Q_{N}^{\text {upper }}(x)=\left(\begin{array}{c}
\frac{1}{2} \\
N+1
\end{array}\right) x^{N+1} .
\end{aligned}
$$

\section{$N$ is Odd, $x$ is Positive}

If $N$ is odd and $x>0$, then $\left(\begin{array}{c}\frac{1}{2} \\ N+1\end{array}\right) x^{N+1}<0$. That gives,

$$
\frac{\partial}{\partial c}\left[\left(\begin{array}{c}
\frac{1}{2} \\
N+1
\end{array}\right) x^{N+1} \frac{1}{(1+c)^{N+\frac{1}{2}}}\right]>0 .
$$

As the derivative is positive, the lower limit is found by inserting the lowest value for $c$, and vice versa. Because $x>0, c$ is constrained by $0<c<x^{\max }$. Hence,

$$
\begin{aligned}
& Q_{N}^{\text {lower }}(x)=\left(\begin{array}{c}
\frac{1}{2} \\
N+1
\end{array}\right) x^{N+1} \\
& Q_{N}^{\text {upper }}(x)=\left(\begin{array}{c}
\frac{1}{2} \\
N+1
\end{array}\right) x^{N+1} \frac{1}{\left(1+x^{\max }\right)^{N+\frac{1}{2}}} .
\end{aligned}
$$

\section{$N$ is Odd, $x$ is Negative}

If $N$ is odd and $x<0$, then $\left(\begin{array}{c}\frac{1}{2} \\ N+1\end{array}\right) x^{N+1}<0$. That gives,

$$
\frac{\partial}{\partial c}\left[\left(\begin{array}{c}
\frac{1}{2} \\
N+1
\end{array}\right) x^{N+1} \frac{1}{(1+c)^{N+\frac{1}{2}}}\right]>0
$$

As the derivative is positive, the lower limit is found by inserting the lower value for $c$, and vice versa. Because $x<0, c$ is constrained by $x^{\mathrm{min}}<c<0$. Hence, 


$$
\begin{aligned}
& Q_{N}^{\text {lower }}(x)=\left(\begin{array}{c}
\frac{1}{2} \\
N+1
\end{array}\right) x^{N+1} \frac{1}{\left(1+x^{\text {min }}\right)^{N+\frac{1}{2}}}, \\
& Q_{N}^{\text {upper }}(x)=\left(\begin{array}{c}
\frac{1}{2} \\
N+1
\end{array}\right) x^{N+1} .
\end{aligned}
$$

Multiplying Eq. 45 with the non-negative constant $\frac{R}{\sin (\alpha)}$ gives

$$
\begin{aligned}
\frac{R}{\sin (\alpha)} Q_{N}^{\text {lower }}(x) & \leq \frac{R}{\sin (\alpha)} Q_{N}(x) \\
& \leq \frac{R}{\sin (\alpha)} Q_{N}^{\text {upper }}(x)
\end{aligned}
$$

Using the same reasoning as in Section 6.1 ensures that Eq. 59 also holds after integration w.r.t. $\theta$, provided that $\theta_{2}>\theta_{1}$. That is

$$
\begin{aligned}
\int_{\theta_{1}}^{\theta_{2}} \frac{R}{\sin (\alpha)} Q_{N}^{\text {lower }}(x) \mathrm{d} \theta & \leq \int_{\theta_{1}}^{\theta_{2}} \frac{R}{\sin (\alpha)} Q_{N}(x) \mathrm{d} \theta \\
& \leq \int_{\theta_{1}}^{\theta_{2}} \frac{R}{\sin (\alpha)} Q_{N}^{\text {upper }}(x) \mathrm{d} \theta
\end{aligned}
$$

Inserting Eq. 41 into Eq. 60 gives the final result

$$
\begin{aligned}
& \int_{\theta_{1}}^{\theta_{2}} \frac{R}{\sin (\alpha)} Q_{N}^{\text {lower }}(x) \mathrm{d} \theta \\
& \leq s\left(\theta_{1}, \theta_{2}\right)-\int_{\theta_{1}}^{\theta_{2}} \frac{R}{\sin (\alpha)} P_{N}(x) \mathrm{d} \theta \\
& \leq \int_{\theta_{1}}^{\theta_{2}} \frac{R}{\sin (\alpha)} Q_{N}^{\text {upper }}(x) \mathrm{d} \theta .
\end{aligned}
$$

Hence, the uppermost and lowermost integrals of Eq. 61 provide lower and upper limits, respectively, for the difference between the true length of a helical cable element and the approached based on Maclaurin series.

The uppermost and lowermost integrals of Eq. 61 can be integrated analytically as $x^{N+1}$ is the only factor depending on $\theta$ in the expressions $Q_{N}^{\text {lower }}(x)$ and $Q_{N}^{\text {upper }}(x)$, where $x$ is defined in Eq. 8. However, note that $Q_{N}^{\text {lower }}(x)$ and $Q_{N}^{\text {upper }}(x)$ are defined differently for $x>0$ and for $x<0$. Hence, the integral must be divided into subintervals if $x$ alters between being positive and being negative within the interval given by the integration limits.

\subsection{Numerical Example of Error Estimation}

This section presents a numerical example of the method of error estimation which is derived in Section 6.3. The numerical values stated in Table 2 are used in the example. The length of a helical element over one half pitch length in the cable's outer arc is calculated, that is, the integration limits are $\theta_{1}=0$ and $\theta_{2}=\pi$.

Table 3 presents the numerical results. The first column shows the order of the Maclaurin polynomial which the calculation is based on. The second column is the length of the helical element after cable bending calculated by the Maclaurin approach derived in this paper. The third and forth columns are the lower and upper limits, respectively, of the estimation error. That is, the third column is the first row of Eq. 61, and the forth column is the third row of this equation. The fifth column is the sum of the second and third columns, while the sixth column is the sum of the second and forth columns. Hence, the true value of the helical element's length, $s\left(\theta_{1}, \theta_{2}\right)$, is ensured to be between the value of the fifth column and the value of the sixth column.

The second column of Table 3 indicates that the calculated length of the helical element converges to a certain value. The most important result of the table is the third and forth columns, which shows the the estimation error decreases rapidly as the order of the Maclaurin polynomial increases. The fifth and sixth columns show that the interval which the true element length is ensured to be within quickly narrows as the polynomial order increases. These results are expected from the proof in Section 6.1.

Table 4 shows numeric integration of Eq. 6 using Simpson's method. This numeric method tends to converge to a certain value as the number of discretization intervals increases. This is similar to the Maclaurin approach which tends to converge for increasing polynomial order as shown in the second column of Table 3. By comparing the two tables, it is shown that the Maclaurin approach and Simpson's method converge to the same value. Simpson's method here serves as a verification that the Maclaurin approach derived in this paper is correct. The main advantage of the Maclaurin approach over Simpson's method is that the former provides an analytical solution of the length integral, which can be used in mechanical analyses of cables and umbilicals.

In Table 4 the error is reduced by a factor of approximately 16 for each time the number of discretization intervals is doubled, expect for from two to four intervals, where the factor is approximately 21 . The calculations of the errors are based on comparison with Simpson's method using $10^{6}$ intervals. Please note that more decimals are used in these calculations than those shown in Table 4. According to Edwards and Penney (1986), the error of Simpson's method is approximately proportional to $\frac{1}{n_{i}^{4}}$, where $n_{i}$ is the number of discretization intervals. Hence, according to this theory, the integration error will decrease by a factor of approximately 16 as the number 
Table 3. Error estimation using the method derived in Section 6.3. Please refer to the main text for explanation.

\begin{tabular}{lrrrrr}
\hline & Calculated length $[\mathrm{m}]$ & Lower error $[\mathrm{m}]$ & Upper error $[\mathrm{m}]$ & Lower length limit $[\mathrm{m}]$ & Upper length limit $[\mathrm{m}]$ \\
\hline$N=1$ & 2.6973895 & -0.0060811 & -0.0046426 & 2.6913083 & 2.6927468 \\
$N=2$ & 2.6913083 & 0.0003238 & 0.0005077 & 2.6916321 & 2.6918160 \\
$N=3$ & 2.6918160 & -0.0000552 & -0.0000294 & 2.6917608 & 2.6917866 \\
$N=4$ & 2.6917608 & 0.0000031 & 0.0000069 & 2.6917639 & 2.6917677 \\
\hline
\end{tabular}

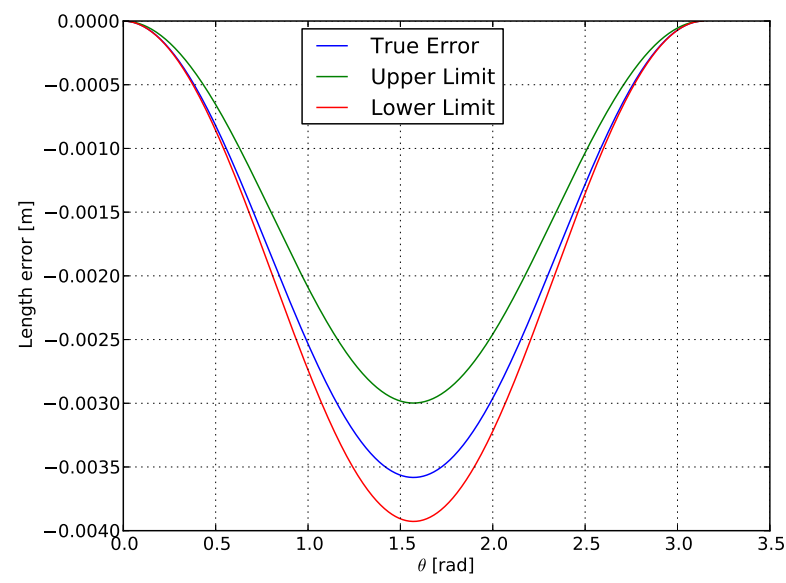

Figure 5. Upper and lower error estimate compared to the true error for $N=1$.

of intervals is doubled, which is in accordance with the observations.

Table 4. Numeric integration of Eq. 6 using Simpson's method.

\begin{tabular}{rr}
\hline Number of intervals & Calculated length $[\mathrm{m}]$ \\
\hline 2 & 2.6990374 \\
4 & 2.6921142 \\
8 & 2.6917874 \\
16 & 2.6917682 \\
32 & 2.6917670 \\
64 & 2.6917669 \\
128 & 2.6917669 \\
\hline
\end{tabular}

Figures 5 - 8 illustrate Table 3 graphically for different values of $N$. In each of the figures, the blue curve represents the difference between the true element length and the element length calculated by the Maclaurin expansion. More precisely, the blue curve is $\frac{\mathrm{d} s}{\mathrm{~d} \theta}-\frac{R}{\sin (\alpha)} P_{N}(x)$. Integrating this term w.r.t. $\theta$ from $\theta_{1}$ to $\theta_{2}$ gives the second line of Eq. 61. That is, the area between the blue curve and the first axis (x-axis) is the difference between the true length of the helical cable element and the approach based on Maclaurin polynomial as derived in the present paper. Areas above the first axis are considered positive, and areas below the first axis are considered negative.

The red and green curves of Figures 5 - 8 represent the

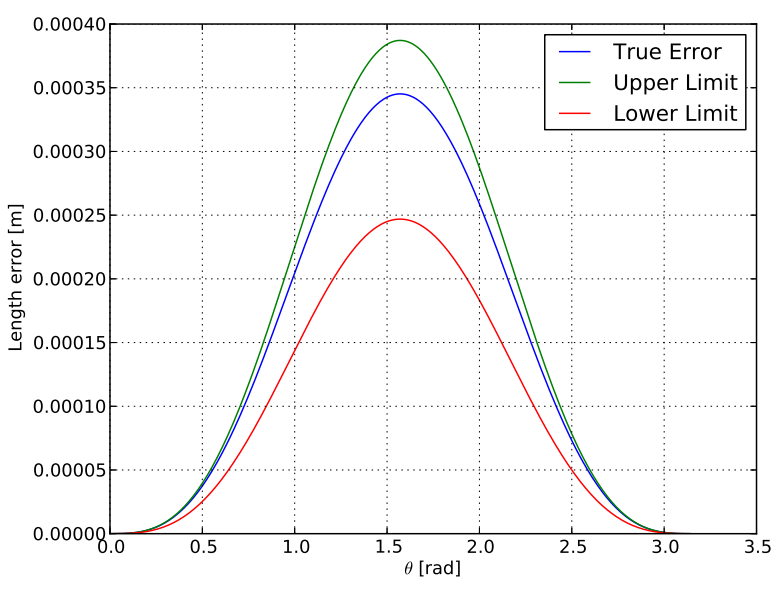

Figure 6. Upper and lower error estimate compared to the true error for $N=2$.

lower and upper limits of the estimation error. More precisely, the red curve is $\frac{R}{\sin (\alpha)} Q_{N}^{\text {lower }}(x)$ and the green curve is $\frac{R}{\sin (\alpha)} Q_{N}^{\text {upper }}(x)$. Integrating these terms w.r.t. $\theta$ from $\theta_{1}$ to $\theta_{2}$ gives the first row and the third row of Eq. 61, respectively. That is, the first row of Eq. 61 is equal to the area between the red curve and the first axis. Similarly, the third row of Eq. 61 is equal to the area between the green curve and the first axis. Also, the areas between the red curve and the first axis, and between the green curve and the first axis, are equal to the values of the third and forth columns of Table 3 , respectively.

In Figures 5 - 8 the blue curve is always above the red curve and below the green curve. Hence, the area between the blue curve and the first axis is larger that the correspond area of the red curve, but smaller than the corresponding area of the green curve, taking into consideration that areas below the first axis are considered negative. Hence, the graphs of the four figures are graphical illustrations of the inequality of Eq. 61 .

Figures 5 - 8 also summarize the main contribution of this paper: The area between the blue curve and the first axis is the error between the true length of the helical element and the calculation based on the Maclaurin polynomial. This area can not be found using analytical integration. However, the blue curve is always above the red curve and below the green curve. Hence, the red and green curves are limits that the blue curve is ensured to be 


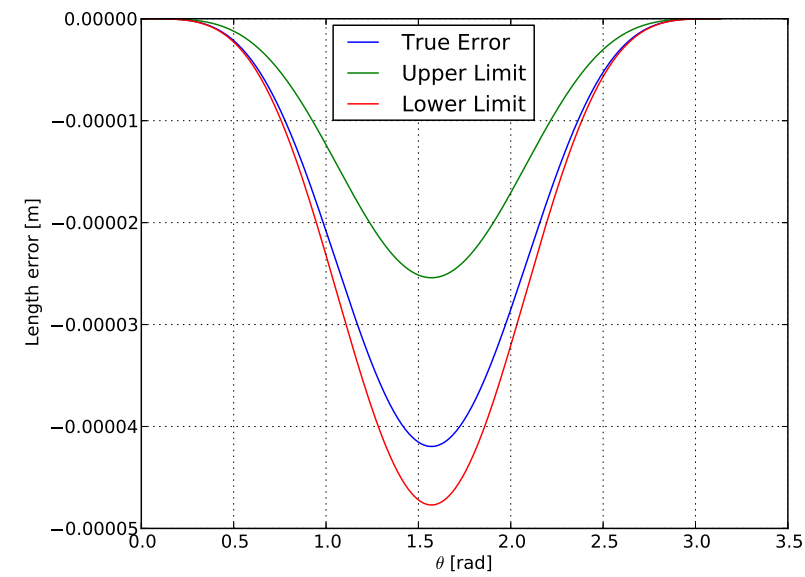

Figure 7. Upper and lower error estimate compared to the true error for $N=3$.

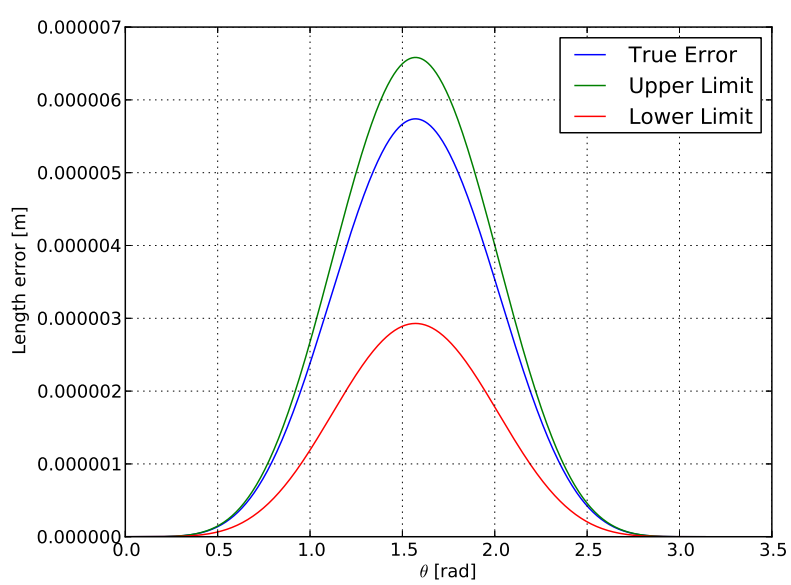

Figure 8. Upper and lower error estimate compared to the true error for $N=4$.

within. The area between the red curve and the first axis, and the area between the green curve and the first axis, can be calculated using analytical integration. Therefore, the latter two areas are lower and upper limits, respectively, that the error of the Maclaurin polynomial approach is ensured to be within. Figures 5 - 8 also show how the error of the Maclaurin polynomial approach decreases as the polynomial order increases: Note the difference between the scales of the second axes (y-axes) in the four figures.

\section{Conclusion}

The present paper derives an expression for the arc lengths of helical cable elements at cable bending. This expression is an integral that to the author's knowledge can not be solved analytically. The integrand of the expression is then approximated by a Maclaurin series expansion, which terms can be integrated analytically.
In order to establish correct strains in the helical cable elements, which is essential for mechanical analyses of the cable, the integrals must be calculated with very high accuracy. An integration error of $0.01 \%$ will in many cases be unacceptable. In this paper it is proved that arbitrary integration accuracy can be achieved by increasing the order of the Maclaurin polynomial. The paper also derives lower and upper limits of the integration error, which is caused by truncation of the Maclaurin series.

The results of the paper are illustrated in a numerical example. The example compares the proposed Maclaurin approach with numeric integration using Simpson's method. The comparison shows that both the Maclaurin approach and Simpson's method perform very well. The Maclaurin approach has the inherent advantage of providing an analytical expression for the integral, which can be used in mechanical analyses of cables and umbilicals.

\section{References}

C. H. Edwards and D. E. Penney. Calculus and analytic geometry - 2nd edition. Prentice-Hall International Ltd., 1986.

J. J. Féret and C. L. Bournazel. Calculation of stresses and slip in structural layers of unbonded flexible pipes. Journal of Offshore Mechanics and Arctic Engineering, 109:263 - 269, 1987.

E. Kebadze. Theoretical modelling of unbonded flexible pipe cross-sections. PhD thesis, South Bank University, 2000.

M. Komperød, B. Konradsen, and R. Slora. Theoretical and practical considerations of helical cable elements subject to end effects at cable bending. In Proceedings of the ASME 2015 34th International Conference on Ocean, Offshore and Arctic Engineering OMAE 2015, 2015.

M. Lutchansky. Axial stress in armor wires of bent submarine cables. Journal of Engineering Industry, 91(3):687 - 693, 1969.

ProofWiki. Binomial theorem/general binomial theorem, 2015. URL www.proofwiki.org/wiki/Binomial_ Theorem/General_Binomial_Theorem.

N. Sødahl, G. Skeie, O. Steinkjær, and A. J. Kalleklev. Efficient fatigue analysis of helix elements in umbilicals and flexible risers. In Proceedings of the ASME 29th International Conference on Ocean, Offshore and Arctic Engineering OMAE 2010, 2010.

G. Skeie, N. Sødahl, and O. Steinkjer. Efficient fatigue analysis of helix elements in umbilicals and flexible risers: Theory and applications. Journal of Applied Mathematics, 2012.

S. Sævik. On Stresses and Fatigue in Flexible Pipes. $\mathrm{PhD}$ thesis, Norwegian Institute of Technology, 1992.

G. B. Thomas, M. D. Weir, and J. Hass. Thomas' calculus global edition - 12th edition. Pearson Education, Inc., 2010. 